\title{
mm-Wave Silicon ICs: Challenges and Opportunities
}

\author{
Invited Paper \\ Ali Hajimiri \\ California Institute of Technology, Pasadena, CA 91125, USA
}

\begin{abstract}
Millimeter-waves offer promising opportunities and interesting challenges to silicon integrated circuit and system designers. These challenges go beyond standard circuit design questions and span a broader range of topics including wave propagation, antenna design, and communication channel capacity limits. It is only meaningful to evaluate the benefits and shortcoming of silicon-based mm-wave integrated circuits in this broader context. This paper reviews some of these issues and presents several solutions to them.
\end{abstract}

\section{Introduction}

Silicon integrated circuits have served as a remarkably unparalleled engine for semiconductor growth in the last half a century [1]-[3]. This exponential growth, often referred to as Moore's law [4], has been sustained to this date, resulting in silicon transistors with cut-off frequencies above $100 \mathrm{GHz}$. While transistors capable of operating at such frequencies have been commonplace in compound semiconductors, the integration of such transistors in silicon offers a qualitative change in the landscape of mm-wave circuit and system design. This is primarily due to the possibility of integrating millions of such transistors on a single die with remarkably high yields at a low incremental cost. Silicon also offers the opportunity to integrate the digital signal processing, self calibration, and self-test features alongside the mm-wave system, while minimizing or even eliminating the high frequency electrical interface to the outside world [5].

While the possibility of making silicon circuits operate at mmwave frequencies has been demonstrated (e.g., [7][10][11]), the full benefits of silicon integration can only be realized by going beyond the direct transfer of techniques and architectures from conventional compound semiconductor approaches to silicon. To take full advantage of what silicon ICs have to offer, one should investigate the system at the highest possible architectural level of abstraction and provide an overall solution keeping in mind the incremental cost of extra transistors and the superior repeatability and matching of silicon integrated components. Approaching system level design in this fashion has led to novel system architectures, a few examples of which can be found in recent literature, such as $[5][6][8][9]$.

Circuit design for mm-wave systems is certainly more than an academic curiosity. Many emerging and existing commercial applications can benefit from cheaper, more efficient, and more compact silicon-based mm-wave solutions. Many of these improvements would invariably be a direct result of the minimization of the number of high-frequency interfaces to the outside world.

Examples of such applications include gigabit wireless LAN (local area networks), point-to-point ultra-high speed communications (e.g., LMDS), and local rebroadcasting of high throughput data sources (e.g., HDTV within a home) on the communication side.

Vehicular ranging and sensing applications (i.e., vehicular radar) can also greatly benefit from high microwave and mm-wave frequency systems in silicon. These systems can offer amenities such as collision avoidance radar, which provides early warning to critical systems such as braking to be applied at full power when needed, as well as autonomous cruise control systems, and full radar imaging of the vehicle surroundings in low visibility situations (e.g., night, fog, or smoke). Other possibilities include self parking, global highway traffic control and management as well as damage minimization in the case of an accident. There are also other vehicular applications such as the "data mule", where cars physically carry large amounts of non-critical data transferred between different units going in different directions, without the need for global wireless coverage.

In addition to these commonplace applications, low-cost compact $\mathrm{mm}$-wave systems with beam forming capabilities can be used as nodes of a sensor network where the formed beam serves both as the sensor and the means of communications.

While there are tremendous opportunities present for mm-wave systems, there are also quite a few challenges in their successful deployment. We will now discuss some of these challenges and opportunities in the subsequent sections:

\section{Propagation}

Perhaps the least discussed issues in IC design circles are the properties of external wave propagation which are often problematic but could provide new opportunities depending on the function of the system in question. In general, the excess propagation loss (in addition to the standard inverse square law dependence) can result in substantial attenuation of the signal. A typical plot of the excess path loss versus frequency is shown in Fig. 1 [12][15]. Quite remarkable is the vertical axis, where the attenuation in $\mathrm{dB}$ is shown on a logarithmic scale itself! The need for "log of a log" to be able to show the attenuation in a meaningful fashion underscores the wide range of change in the excess path loss at different frequencies. The path loss is particularly large at some frequencies due to the absorption lines of certain elements (e.g., the oxygen absorption at $60 \mathrm{GHz}$ results in an excess path loss of $\sim 20 \mathrm{~dB} / \mathrm{km}$ compared to $\sim 0.4 \mathrm{~dB} / \mathrm{km}$ at $94 \mathrm{GHz}$ ). This loss is exacerbated further by humidity and precipitation with an additional attenuation factor determined by the level of precipitation, as shown in Fig. 2 [12]-[15]. Note that the attenuation levels given in Fig. 2 are in excess of those in Fig. 1. This additional loss is of particular interest in outdoor applications, such as vehicular radar and point-to-point communication.

Propagation attenuation is not purely problematic, however. The added loss at frequencies such as $24 \mathrm{GHz}^{1}$ and $60 \mathrm{GHz}$ can result in a faster drop in the signal power resulting in potentially higher frequency reuse ratio for a cellular implementation, where the same frequency can be used nearby by a different base station.

The propagation properties of mm-waves are closer to their yet higher frequency electromagnetic siblings, namely, visible light

1. The mm-wave technically referred to frequencies in the $30 \mathrm{GHz}-300 \mathrm{GHz}$ range whose free space wavelength is between $1 \mathrm{~mm}$ and $1 \mathrm{~cm}$. However, due to the nature of the applications at $24 \mathrm{GHz}$ and the close proximity to the edge of the mm-wave frequencies, we will treat it in a similar fashion. 


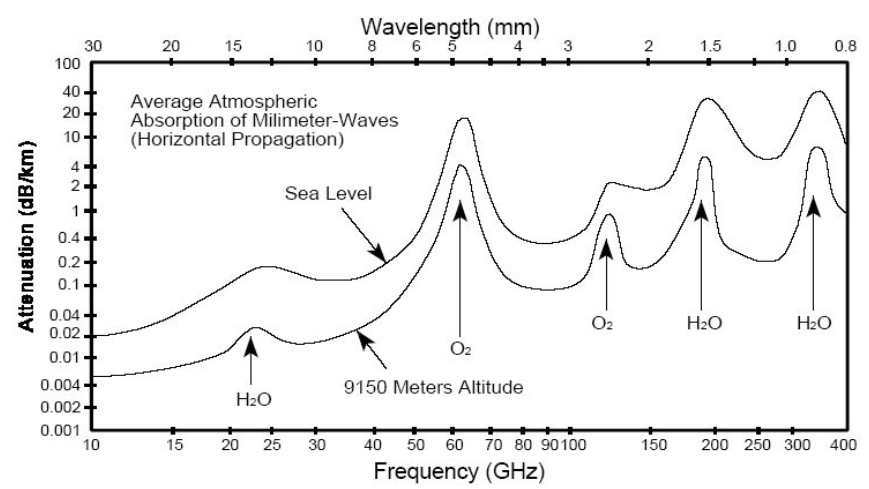

Fig. 1 Atmospheric absorption vs. frequency [12].

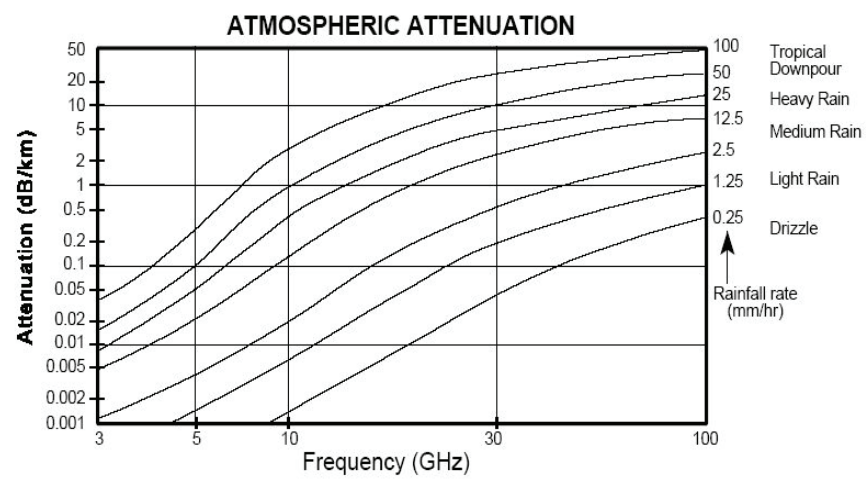

Fig. 2 Added absorption due to precipitation vs. frequency [15].

than are the more traditionally considered lower frequency RF signals. The behavior is more line-of-sight and there is less discernible diffraction compared to lower RF frequencies. This makes them particularly suitable for compact beam forming applications where a collimated beam of the signal can be transmitted in a desirable direction, for instance by using a phased array as discussed later. This is also particularly useful in wireless communications where the same frequency band can be used by two users in close proximity of each other as long as they are not on the same line of sight, which can be interpreted as an effective channel reuse ratio greater than unity.

The mm-waves also experience a larger loss upon reflection off of typical surfaces. This could pose a challenge to obtain reliable wireless coverage in the absence of line-of-sight view of the transmitter, but at the same time it produces a smaller delay spread in the channel impulse response [16]. This corresponds to a channel with smaller multi-path dispersion, which can in turn lead to a higher effective channel capacity.

\section{Antennas}

The most commonly used antennas are passive and as such do not generate power. The directivity of an antenna is a measure of how much it can focus the energy in a particular direction at the expense of lower signal transmission (and hence reception due to reciprocity) in other directions. The reference value of comparison is an omni-directional (isotropic) antenna that transmits (and receives) power equally in all directions. Thus the directivity of an antenna is measured in $\mathrm{dBi}(\mathrm{dB}$ with respect to isotropic). (Fig. 3 ). Of course, directivity comes at the cost of spatial coverage since higher gain antennas must have higher directivity due to conservation of energy. If the antenna has internal energy loss, (e.g., due to ohmic resistance of the conductor used) part of the

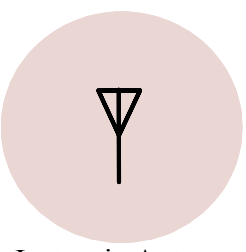

Isotropic Antenna

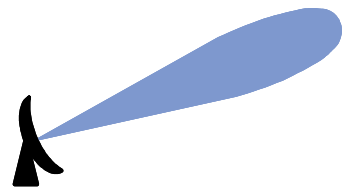

Directional Antenna
Fig. 3 A low gain isotropic antenna contrasted with a directional

energy entering the antenna terminal would turn into heat and the rest of it will turn into electromagnetic (EM) radiated energy. The antenna gain is the product of this energy efficiency and its directivity. Since in a lossless antenna the total radiated energy over all solid angles should be the same as that of an isotropic antenna over a unit sphere, we have:

$$
\oint G d \Omega=4 \pi
$$

where $G=G(\theta, \phi)$ is the gain as a function of direction. One can define an effective area for an antenna as a function of direction $(\theta$ and $\phi)$ as:

$$
A(\theta, \phi)=\frac{P_{r}}{S(\theta, \phi)}
$$

where $P_{r}$ is the total received power and $S(\theta, \phi)$ is the incident power density in that direction. Obviously, directional antennas will have different areas (effective cross-section) when viewed from different directions. The antenna appears large in the direction of maximum gain and small when viewed from other directions. The antenna theorem [17] states:

$$
\oint A d \Omega=\lambda^{2}
$$

where $\lambda$ is the wavelength in the direction of interest. This indicates that, for example, the effective area of an isotropic antenna drops as the square of frequency. This has an important corollary, namely, for a given directionality, a given antenna looks smaller at higher frequencies and hence it collects less power. Equivalently, we could say that the antenna has to become more directional at higher frequencies, if we wish to maintain the same collection area. This poses a challenge that could be turned into an opportunity by using phased arrays.

If we were to use a single relatively isotropic antenna for wireless communications, the antenna will collect less power at higher frequencies, making it more difficult to maintain a desired signal-tonoise ratio. Now, one could try to use a more directional antenna to recover the lost gain, but this solution will be limited to cases where the relative locations and orientations of neither transmitter nor receiver change rapidly (e.g., fixed point-to-point communications). One could try to solve this problem by using multiple antennas to increase the effective collection area, which again results in an increase in the directivity. However, by using phased arrays we could change the direction of the beam electronically and would hence effectively be able to cover a larger area. We will perform a more quantitative analysis of the impact of the antenna area reduction with frequency on channel capacity in a later section.

Another important parameter of an antenna is its radiation resistance. An antenna will present an impedance to the driving port which typically has reactive (imaginary) and resistive (real) parts, 


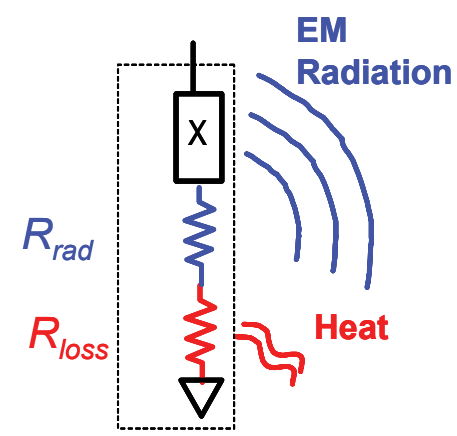

Fig. 4 Electrical equivalent model of an antenna.

as in Fig. 4. The resistive part can be divided into two components, the radiation and the loss resistances. The loss resistance is due to the energy loss mechanism that converts energy to heat, such as ohmic loss in the antenna conductor. It is a parasitic element that should be minimized, as will be seen shortly. On the other hand, the radiation resistance is the desired part which models the energy converted from the electrical domain to electromagnetic radiation power leaving the system. Obviously, the reason that this energy conversion is modeled as a resistor is that the energy has been "lost" in the electrical domain ${ }^{2}$. It should be clear that the radiation resistance has nothing to do with the ohmic resistance of the antenna conductor, and even if it were made out of a superconductor, the radiation resistance would have remained the same.

The power efficiency of an antenna is the ratio of the electromagnetic energy radiated by it to the electrical energy entering it. This ratio is related to the radiation resistance, $R_{\text {rad }}$, and the loss resistance, $R_{\text {loss }}$ in the following fashion:

$$
\eta \equiv \frac{P_{\text {rad }}}{P_{\text {elec }}}=\frac{R_{\text {rad }}}{R_{\text {rad }}+R_{\text {loss }}}
$$

We will see in the on-chip antennas section that maintaining a high $R_{\text {rad }} / R_{\text {loss }}$ ratio is a challenge in silicon integrated antennas.

\section{Silicon Integration}

Despite numerous advantages to integration of mm-wave systems on a silicon substrate mentioned earlier, there are certain challenges for full integration of mm-wave systems on silicon, as discussed in this section.

Various energy loss mechanisms become important at mm-wave frequencies. Unlike most other compound semiconductors, silicon substrate is conductive in modern integrated circuits (with a typical substrate resistivity of approximately $10 \Omega \mathrm{cm}$ for bulk processes). This conductivity causes energy loss due to magnetically induced eddy currents. It can also result in a considerable energy loss in addition to the energy losses in metallic conductors if the antenna is to be placed on chip, as will be discussed later.

Passive devices also suffer from energy loss due to their finite conductivity. The ohmic energy loss can be significantly more important at mm-waves because of the small skin depth (e.g., the skin depth of copper at $60 \mathrm{GHz}$ is approximately $0.3 \mu \mathrm{m}$ ). As most of the current is carried on the surface, the surface roughness that

2. An analogous situation arises in a loudspeaker where the acoustic impedance is determined by the energy conversion from the electrical domain to acoustic domain.

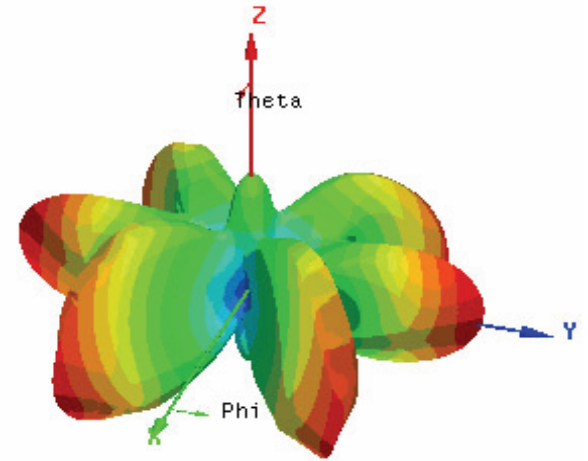

Fig. 5 An example of substrate mode radiating in undesirable directions for a rectangular substrate

can be quite large in modern CMP based processes becomes important. The additional "fill" and "cheese" rules for wide metal strips also degrade the current handling capabilities of the metal lines.

An equally important effect that is given little attention in the IC design community is the high dielectric constant of most semiconductor substrates, including silicon (e.g., $\varepsilon_{r}=11.7$ for silicon). The rectangular silicon substrate forms a dielectric waveguide that can sustain propagating modes depending on its physical dimensions. Unfortunately, many of the natural modes for a typical silicon die size fall right in the middle of the mm-wave bands of interest. This in turn creates an alternative mechanism for energy loss for on chip components, where energy could easily leak into the substrate modes and be dissipated by ohmic loss within the substrate, or even worse, be radiated in undesirable directions [5] (Fig. 5.). Also, energy can be easily coupled back into other on-chip components creating parasitic, yet potentially strong, coupling between seemingly unrelated elements on the same chip. Special care must be paid to the overall design to avoid such coupling.

For an on-chip antenna, the high dielectric constant of the substrate causes a considerable portion of the electromagnetic energy to be sucked into the substrate as opposed to being radiated into the air from the top side [18][5]. In the case of silicon, the impact of this effect is further exacerbated by the conductivity of the substrate. This is a very important issue when trying to integrate the antennas with the rest of the chip, as will be discussed further in the section dealing with on-chip antennas.

Power generation in small-feature size silicon technologies also presents a very serious challenge, mostly due to the lower breakdown voltages resulting from the scaling process and the shrinking of the depletion regions in the transistors. This necessitates the use of a lower supply voltage. Unfortunately, this is in direct conflict with the maximum mm-wave power that can be generated using conventional power amplifier techniques, necessitating the use of parallel structures and novel power combing approaches as described in [19][20].

Obviously, the modeling of the transistors (as well as the passive devices) becomes more challenging at these higher frequencies. The smaller parasitic components within the models are more prone to error, and hence special attention must be paid to guarantee reliable results, especially in the presence of process variations and environmental changes. 


\section{High Frequency Interface}

Even when all the challenges of making mm-wave integrated circuits in silicon have been overcome, we still need to be concerned about the mm-wave interface to the outside world. External connections to the chip need to be rethought completely. As an example, consider the case, when we connect the mm-wave input (or output) of the chip to a perfectly matched transmission line on a adjacent PCB using a wirebond. Modeling a $1 \mathrm{~mm}$ long wirebond as a $1 \mathrm{nH}$ inductor ${ }^{3}$ with a $Q$ of 30 we find that our connection has an impedance of approximately $15 \Omega+j 460 \Omega$ at $77 \mathrm{GHz}$, making it very difficult to achieve a proper impedance match. Furthermore, such an element can easily turn into an unintended antenna at mm-wave frequencies, creating very serious problems at the system level. Even a solder ball in flip-chip technology presents a non-trivial impedance to match to the outside world. Reported results based on direct wafer probing of the die entirely ignore this challenge. If one tries to make the interface with wirebond, every effort should be made to make it into a structure emulating a transmission line, as in [9]. On the other hand, these interface challenges make the totally-integrated solutions with on-chip antenna much more attractive by offering a solution where the high frequency electrical interface to the outside world is completely eliminated and replaced by an electromagnetic radiating interface. An example of such an approach can be found in [5][6].

All of the above considerations demonstrate the enormous opportunities and important challenges in the area of mm-wave integrated circuits. It is clear that the direct application of microwave approaches to design of silicon-based integrated circuits, is neither efficient nor optimum, as these techniques are fundamentally based on the underlying assumption of using a small number of well-characterized transistors. At the same time, direct application of lower frequency analog techniques to this problem would not be particularly successful either, as in the absence of proper treatment of the complex EM issues involved in such systems, the designers will not be able to come up with an implementation that closely matches the expected results. Thus, it is clear that to take full advantage of this significant opportunity, one needs to start at the system architecture level and devise new methodologies and architectures along the way.

\section{Capacity Limits}

Shannon's theorem predicts that the maximum error-free bit rate of a channel with a bandwidth of $B W$ and a signal-to-noise ratio of $\mathrm{S} / N$ is given by

$$
R_{B}=B W \cdot \log _{2}\left(1+\frac{S}{N}\right) .
$$

Although at first glance it may appear that going to higher frequencies automatically results in higher bit rates due to greater available bandwidths, the signal-to-noise term's degradation at very high frequencies diminishes this improvement.

To obtain a more realistic picture, we need to take several effects into account. Assuming a low directivity single-antenna to be able to communicate in different directions, we expect the effective antenna area to be similar in different directions and proportional to $\lambda^{2}$. Also, let us assume a BW that grows linearly with the center frequency. In our example, let us assume that the BW

3. This assumption is not explicitly correct at mm-wave frequencies as a $1 \mathrm{~mm}$ wirebond is comparable in dimensions to the wavelength and its port impedance is a function of the return path and the termination that in general should be determined via electromagnetic simulations. Nonetheless, this example is to illustrate the impact of the smallest of parasitic components.

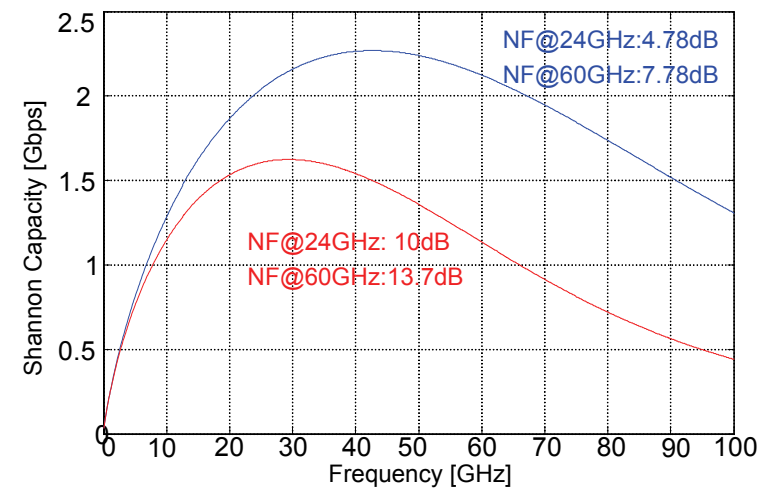

Fig. 6 Shannon capacity vs. frequency.

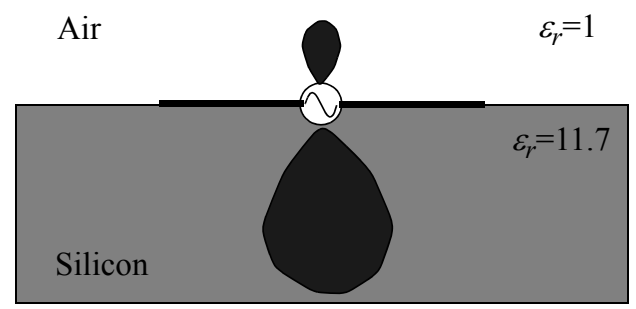

Fig. 7 Dipole antenna at the air-silicon boundary

is about $1 \%$ of the center frequency, i.e., $B W \approx f_{0} / 100$. Assuming a distance of $10 \mathrm{~m}$ and that the noise figure degrades linearly with frequency ${ }^{4}$, we can calculate the Shannon capacity as a function of frequency, as plotted in Fig. 6 for two different initial $N F$ values. It is noteworthy that the Shannon capacity approaches a maximum and goes back down, mainly due to lower collected signal power due to the lower antenna collection area and worse receiver noise performance.

Fig. 6 is a clear indication that to be able to take full advantage of mm-wave frequencies, we must consider multiple antennas with electronic steering capability to increase the collection area, while maintaining good spatial coverage by steering the beam in the desired direction without any mechanical movement.

\section{On Chip Antennas}

On-chip antennas make it possible for the input and output signals to be radiated directly into and out of the chip with no need for an electrical interface at mm-wave frequencies. However, successful implementation of an on-chip antenna with reasonable gain and efficiency is a non-trivial task primarily due to the high dielectric constant and conductivity of the substrate.

To demonstrate this point more clearly, let us consider a dipole antenna placed at the interface of air and a semi-infinite region of a dielectric material with a relative permittivity of $\varepsilon_{\mathrm{r}}$, as shown in Fig. 7. The dielectric presents a lower electromagnetic impedance than the air and as a result more of the radiated electromagnetic power will end up in the dielectric. Fig. 8 shows the percentage of the power going in each direction as a function of the $\varepsilon_{\mathrm{r}}[18][5]$. As can be seen, for silicon with an $\varepsilon_{\mathrm{r}}$ of approximately 11.7 , more than $95 \%$ of the power will end up going into the silicon, as opposed to the air.

4. In this calculation it is assumed that we can generate power at higher frequencies with the same facility as lower frequencies. This assumption is clearly not accurate. Nonetheless inclusion of any degradation of power vs. frequency will only make the peak in capacity occur at a lower frequency, while maintaining the general behavior of the curve. 


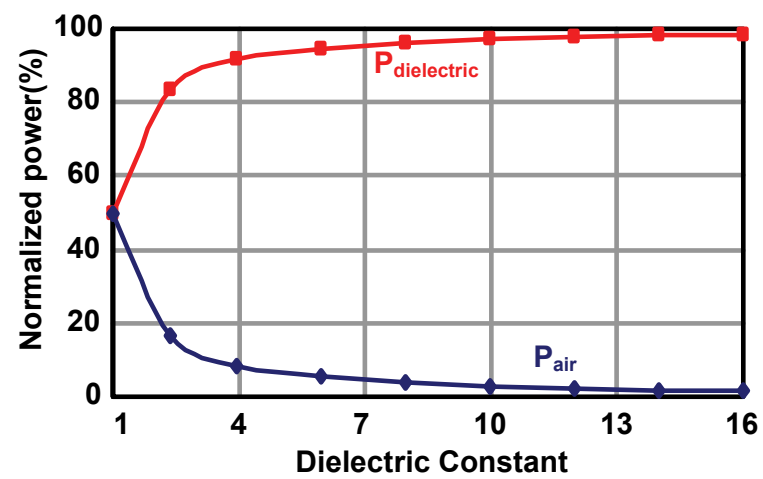

Fig. 8 Percentage of the radiated power into the air and dielectric vs. relative permittivity of the dielectric for a dipole antenna at the boundary [5].

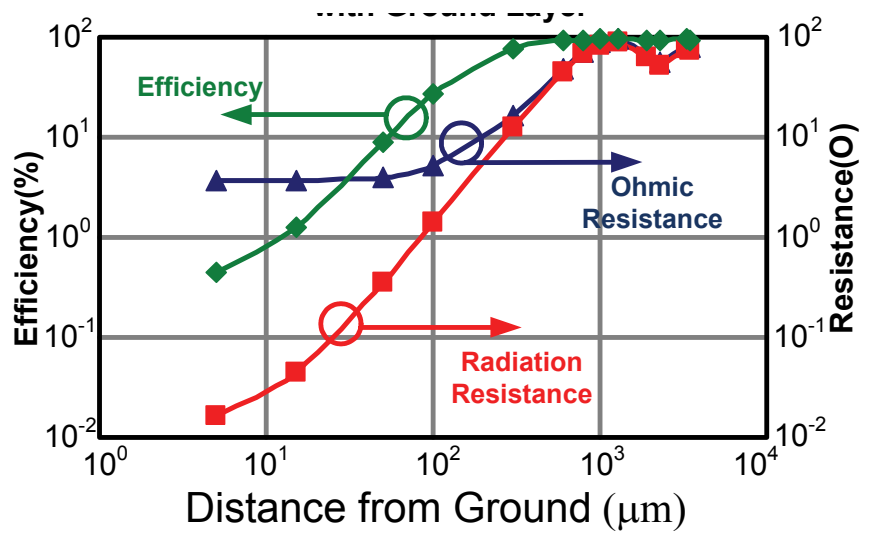

Fig. 9 The efficiency, radiation and loss resistance of a dipole vs. spacing from the ground plane.

To avoid this problem, one may decide to use a ground plane in the lowest metal layer (e.g., M1) while placing the antenna in the top-most layer. While this may sound appealing initially, is not very practical due to the typical spacing between the top and bottom metal layers (e.g., in the $10-15 \mu \mathrm{m}$ range in today's technologies). If one were to attempt this approach, the resulting antenna would have such a small radiation resistance due to the close proximity of the ground plane that its efficiency would be extremely low (e.g., $<1 \%$ ). The trade-off between these different parameters is shown in Fig. 9. [5].

Placing the ground plane directly underneath the substrate does not improve upon this situation. Although the spacing may be high enough for the radiation resistance to become acceptably large, the entire substrate will act as a waveguide with multiple propagation modes, some of which will typically fall very close the frequency of interest. The energy does get coupled into these modes where part of it is lost to heat in the conductive substrate and the remainder is radiated in undesirable directions, as shown in Fig. 5.

One approach to solve this problem is to take advantage of the energy coupling into the substrate and radiating it from the backside of the chip. To do this, we must suppress the substrate modes

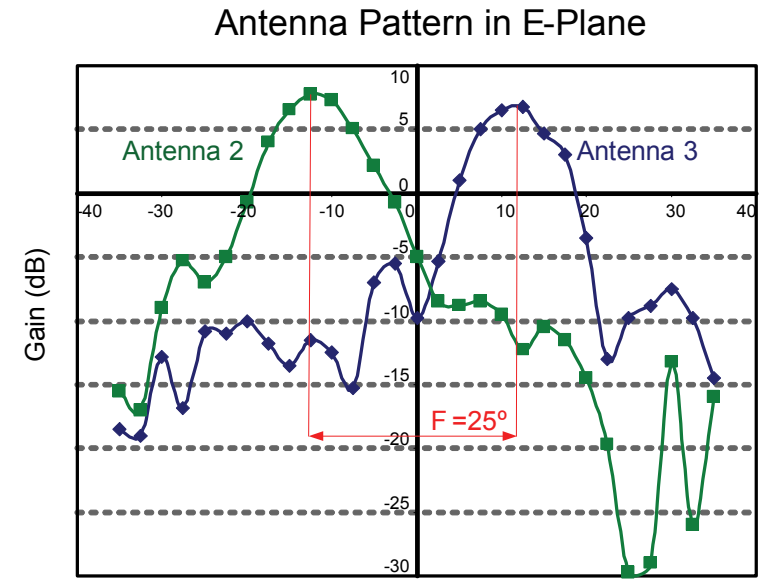

Angle (deg)

Fig. 10 Measured patterns of two adjacent antennas on a silicon chip with a backside lens.

and direct the energy into the desired radiative mode. This can be achieved by changing the "shape" of the substrate electromagnetically.

One way to do this is to use a dielectric lens [18] on the backside of the chip which couples the EM energy into a dominant radiative mode [21]. To reduce the antenna metal loss, three bottom layers are connected in parallel with vias to form the dipole antennas as close to the substrate as possible. To reduce the substrate loss, the fabricated chip is thinned down to $100 \mu \mathrm{m}$. Due to the layout constraints, antennas are placed at the chip edge and to maintain a uniform dielectric constant substrate underneath the antennas, an undoped silicon slab with the same thickness of silicon chip is abutted to the chip. For mechanical stability, a 500um silicon wafer is used to hold the chip and the silicon lens is attached to the backside of the undoped wafer. A 2-axis spherical far field measurement technique is utilized to measure the radiation pattern while a W-band horn antenna is used to irradiate the integrated dipoles. The 3-D measured patterns of two middle antennas are shown in Fig. 10. A maximum peak gain of about $+8 \mathrm{dBi}$ is achieved in this measurement. As seen in Figure 4, the peaks of two antennas occur at the two different directions. The chip requires no high frequency electrical connection to the outside world.

\section{Integrated Phased Arrays}

Integration of a complete phased array system in silicon results in substantial improvements in cost, size, and reliability. It also offers various opportunities to perform on-chip signal processing and conditioning without having to go off-chip, leading to additional savings in cost and power. The multiple signal paths, operating in harmony, provide benefits at the system and circuit level.

Multiple antenna phased-arrays can be used to imitate a directional antenna whose bearing can be controlled electronically [22]-[28]. This electronic steering makes it possible to emulate antenna properties such as gain and directionality, while eliminating the need for continuous mechanical reorientation of the actual 


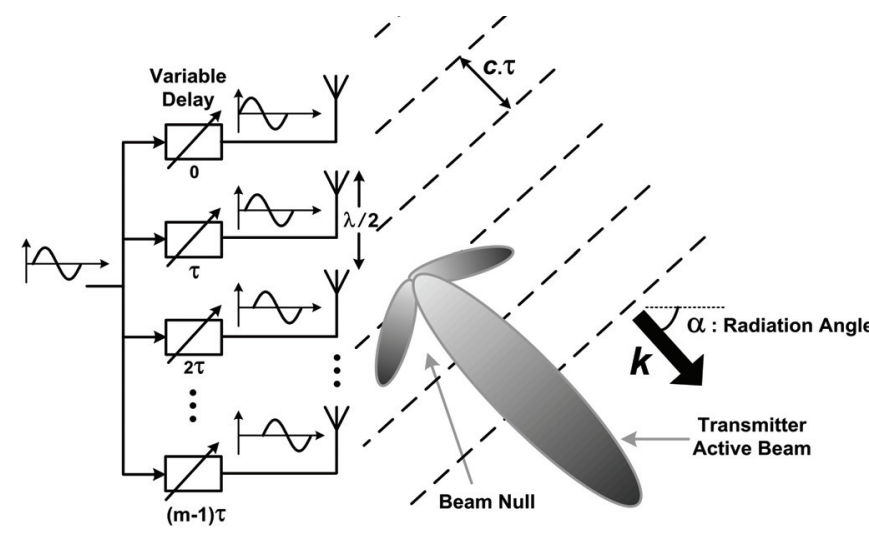

Fig. 11 A phased array transmitter.

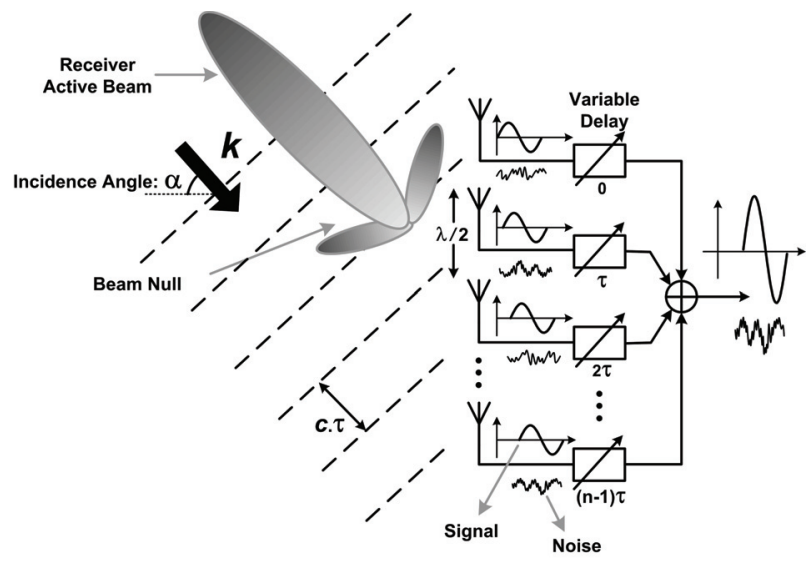

Fig. 12 A phased array receiver.

antennas (Fig. 11). Additionally, the parallel nature of a phased array antenna transceiver alleviates the power handling and noise requirements for individual active devices used in the array. This makes the system more robust to the failure of individual components. In the past, such systems have been implemented using a large number of microwave modules, adding to their cost and manufacturing complexity [26][27].

A phased-array transmitter or receiver consists of several signal paths each connected to a separate antenna. The antenna elements of the array can be arranged in different spatial configurations [24]. The array can be formed in one, two, or even three dimensions, with one or a two dimensional array being more common.

The principle of operation of a phased-array is similar for both receivers and transmitters. In a phased-array receiver, the radiated signal arrives at different times at each of the spatially separated antennas. The difference in the time of arrival of the signal at different antennas depends upon the angle of incidence and the spacing between the antennas. As shown in Fig. 12, an ideal phased-array receiver compensates for the time delay difference between the signals from different antennas and combines the signals coherently to enhance the reception from the desired direction(s), while rejecting emissions from other directions. Similarly, in a phased-array transmitter, the signals in different elements are delayed by different amounts so that the outgoing signals add up coherently only in the desired direction(s). Incoherent addition of the signals in other directions results in lower radiated power in those directions. Thus in a phased-array based system, the transmitter generates less interference in receivers that are not targeted. Furthermore, the receiver is also capable of nulling out some interferers as long as they do not originate from the same direction as the signal. Additionally, for a given power level at the receiver, the power that has to be generated is lower in a phased-array transmitter than in an isotropic transmitter.

In a transmitter with $n$ elements, if each element radiates $P$ Watts, the total power that will be seen at the receiver in the desired direction is $n^{2} P$ Watts. The $n^{2}$ improvement comes from the coherent addition of the signals in amplitude in the desired direction. For example, in a four element transmitter, the total power radiated in the beam direction is $12 \mathrm{~dB}$ higher than the power radiated by each element.

In receivers, the advantages of a phased array include better sensitivity and higher interference rejection capabilities. For a given receiver sensitivity, the output $S N R$ sets an upper limit on the noise figure of the receiver. The noise figure, $N F$, is defined as the ratio of the total output noise power to the output noise power caused only by the source [29]. In an actual phased array receiver implementation, compared to the output $S N R$ of a single-path receiver, the output SNR of the array is improved by a factor between $n$ and $n^{2}$ depending on the noise and gain contribution of different stages [8][9]. For instance, if the noise from the antennas is uncorrelated, an 8-path phased-array can improve the receiver sensitivity by $9 \mathrm{~dB}$.

Thus, in a system based on phased arrays at the transmitter and receiver, the higher SNR and lower interference increases channel capacity. Furthermore, the directivity of the transmit-receive pairs can result in higher frequency reuse ratios, leading to higher network capacity.

For narrowband systems, the true-time delay necessary in each element of a phased-array can be approximated by a phase-shift. This approximation leads to some signal dispersion, due to the non-constant group delay, which increases as the bandwidth of the signal increases. This dispersion translates to a higher BER in communication systems and lower resolution in radar systems [8].

The phase shift necessary in each element of a phased-array can be achieved at RF, at baseband/IF or in the LO path. In integrated implementations, there are several advantages to using LO path phase shifting, as the gain in each element of the transmitter or receiver is less sensitive to the amplitude variations at the LO ports of the mixers [8][9].

The various phases of the LO necessary in the LO-phase shifting approach can be generated in a central fashion by generating all the necessary phases at one place (e.g, a multi-phase oscillator), as in [8][9], or in a decentralized fashion by distributing only one phase of the LO signal and generating the remaining phases locally using a phase rotator for each LO path, similar to [5][6].

A fully integrated transceiver 4-element phased array transceiver with on-chip antennas has been designed and fabricated in a $0.13 \mu \mathrm{m}$ SiGe BiCMOS process [5][6]. The receiver consists of the complete down-conversion path with low-noise amplifier (LNA), frequency synthesizer, phase rotators, combining amplifiers, and on-chip dipole antennas. A distributed active combining amplifier at an IF of $26 \mathrm{GHz}$ is used to perform the signal combining. A 52-GHz first $\mathrm{LO}$ is generated on chip and is routed to different elements, where it can be phase shifted locally by the phase rotators. The local LO-path phase-shifting scheme enables a robust distribution network that scales well with increasing frequency and number of elements while providing high-resolution 


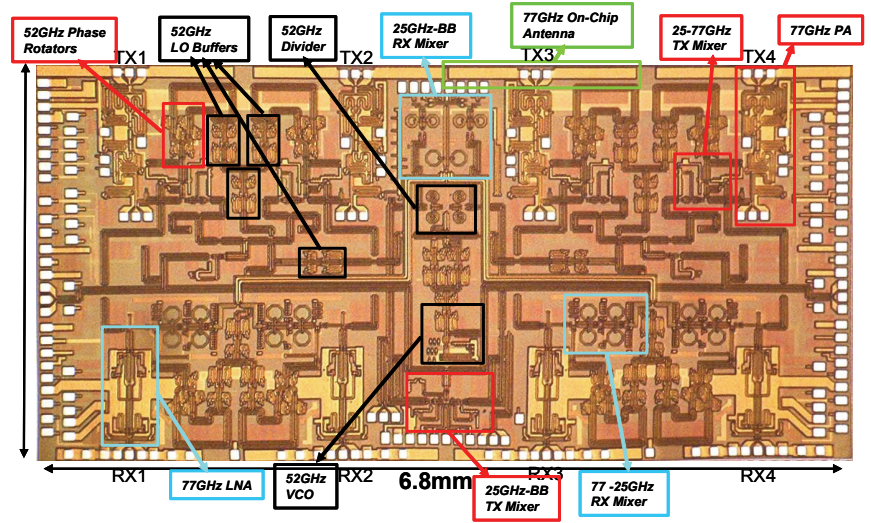

Fig. 13 Die photo of the phased-array transceiver with on-chip antennas

phase shifts. Measurements indicate a single-element LNA gain of $23 \mathrm{~dB}$ and a noise figure of $6.0 \mathrm{~dB}$. Each of the four receive paths has a gain of $37 \mathrm{~dB}$ and a single-path overall noise figure of $8.0 \mathrm{~dB}$. Each on-chip antenna has a gain of $+8 \mathrm{dBi}$. Each element of the 2-step upconversion transmitter generates $+12.5 \mathrm{dBm}$ of output power at $77 \mathrm{GHz}$ with a bandwidth of $2.5 \mathrm{GHz}$ leading to a 4-element effective isotropic radiated power (EIRP) of $24.5 \mathrm{dBm}$. Each on-chip PA has a maximum saturated power of $+17.5 \mathrm{dBm}$ at $77 \mathrm{GHz}$. The entire phased array transceiver occupies an area of $3.8 \mathrm{~mm} \times 6.8 \mathrm{~mm}$, as shown in the die photo of Fig. 13,

\section{Acknowledgments}

The author would like to thank the contributions of A. Babakhani, A. Natarajan, Y. Wang, and H. Wang of Caltech and Dr. A. Komijani, Dr. X. Guan, Prof. H. Hashemi, Prof. J. Buckwalter formerly of Caltech for the numerous contribution to Caltech's mm-wave activity. The authors also would like to thank E. Keehr, F. Bohn, and A. Babakhani for helpful comments on this manuscript. We have benefitted from the support of Caltech's Lee Center for Advance Networking, National Science Foundation, and DARPA Trusted Foundry Program.

\section{References}

[1] J. S. Kilby, United States Patent Number 3,138,743, Filed Feb. 6, 1959, Granted June 23, 1964.

[2] J. S. Kilby, United States Patent Number 3,261,081, Filed Feb. 6, 1959, Granted July 19, 1966.

[3] R. F. Stewart, United States Patent Number 3,138,747, Filed Feb. 12, 1959, Granted June 23, 1964.

[4] G.E. Moore, "Cramming more components onto integrated circuits," Electronics, vol. 38, No. 8, pp. 114-117, April 1965.

[5] A Babakhani, X Guan, A Komijani, A Natarajan, and A. Hajimiri, "A 77-GHz Phased-Array Transceiver with On-Chip antennas in silicon: Receiver and Antennas," IEEE J. SolidState Circuits, vol. 41, no. 12, pp. 2795-2806, Dec. 2006.

[6] A Natarajan, A Komijani, X Guan, A Babakhani, and A. Hajimiri, "A 77-GHz Phased-Array Transceiver with On-Chip antennas in silicon: Transmitter and Local LO-Path Phase Shifting," IEEE J. Solid-State Circuits, vol. 41, no. 12, pp. 28072819, Dec. 2006
[7] X. Guan and A. Hajimiri, "A 24-GHz CMOS front-end," IEEE J. Solid-State Circuits, vol. 39, no. 2, pp. 368-373, Feb. 2003.

[8] A. Hajimiri, A. Komijani, A. Natarajan, X. Guan, H. Hashemi, "Phased array systems in silicon," IEEE Communications Magazine, vol. 42, Issue 8, pp. 122-130, Aug. 2004.

[9] H. Hashemi, X. Guan, A. Komijani, and A. Hajimiri, "A 24GHz SiGe phased-array receiver - LO phase shifting approach," IEEE Trans. Microwave Theory Tech, vol. 53, Issue 2, pp. 614626, Feb. 2005.

[10] C. Doan, S. Emami, A. Niknejad, and R. Brodersen, "Millimeter Wave CMOS Design," IEEE J. Solid-State Circuits, vol. 40, no. 1, pp. 144-155, Jan. 2005.

[11] B. Floyd, s. Reynolds, U. Pfeiffer, T. Zwick, T. Beukema, and B. Gaucher, "SiGe Bipolar Transceiver Circuits Operating at $60 \mathrm{GHz}, "$ IEEE J. Solid-State Circuits, vol. 40, no. 1, pp. 156167, Jan. 2005

[12] CCIR Doc. Rep. 719-3,"Attenuation by Atmospheric Gases," ITU 1990.

[13] L.J. Ippolito, "Propagation Effects Handbook for Satellite Systems Design," NASA Doc. 1082(4), Feb. 1989, ch. 3 and 6 passim.

[14] E.K. Smith, "Centimeter and Millimeter Wave Attenuation and Brightness Temperature due to Atmospheric Oxygen and Water Vapor," Radio Science, Vol. 17, Nov.-Dec. 1982, pp. 1455-1464.

[15] Federal Communications Commission, "Millimeter Wave Propagation: Spectrum Management Implications," Bulletin Number 70, July, 1997

[16] D. Lu and D. Rutledge, "Investigation of Indoor Radio Channel from $2.4 \mathrm{GHz}$ to $24 \mathrm{GHz}$," IEEE AP-S Int. Symp. Dig. Papers, June 2003, pp. 134-137

[17] J. D. Kraus and R. J. Marhefka Antennas, McGraw Hill, 2001.

[18] D. B. Rutledge, et al., "Integrated-circuit antennas," Infrared and Millimeter-Waves, K. J. Button, Ed. New York: Academic, 1983, vol. 10, pp. 1-90.

[19] I. Aoki, S.D. Kee, D.B. Rutledge, and A. Hajimiri, "Fully integrated CMOS power amplifier design using the distributed active-transformer architecture," IEEE J. Solid-State Circuits, vol. 37 , no. 3 , pp. 371-383, March 2002

[20] E. Afshari, H. Bhat, X. Li, A. Hajimiri, "Electrical funnel: A broadband signal combining method," Dig. International SolidState circuits Conference, Feb. 2006.

[21] A. Babakhani and A. Hajimiri, "mm-Wave Phased Arrays in Silicon with Integrated Antennas," Proc. Antenna and Propagation Symposium, June 2007.

[22] V. Aulock, W. H., "Properties of phased arrays," Proc. IRE, vol. 48, No. 10, pp. 1715-1728, Oct. 1960.

[23] R. C. Hansen, Ed., Significant Phased Array Papers. Norwood, MA: Artech House, 1973.

[24] R. S. Elliott, Antenna Theory and Design. Englewood Cliffs, NJ: Prentice-Hall, 1981.

[25] M. Golio, Ed., The RF and Microwave Handbook, Session 6.9, CRC Press LLC, 2000.

[26] D. Parker, D. C. Zimmermann, "Phased arrays - part I: theory and architectures," IEEE Trans. Microwave Theory Tech, vol. 50, No. 3, pp 678-687, Mar. 2002.

[27] D. Parker, D. C. Zimmermann, "Phased-arrays - part II: implementations, applications, and future trends," IEEE Trans. Microwave Theory Tech., vol. 50, No. 3, pp 688-698, Mar. 2002.

[28] B. Kane, L. Geis, M. Wyatt, D. Copeland, and J. Mogensen, "Smart Phased Array SoCs: A Novel Application for Advanced SiGe HBT BiCMOS Technology," Proc. of IEEE.

[29] "IRE standards on electron tubes: Definition of terms," Proc. IRE, vol. 45, pp. 983-1010, July 1957. 57 IRE 7. S2. 